\title{
SELF-ASSESSMENT AND OBSERVER RATING OF EMPATHETIC COMMUNICATION IN PHARMACY STUDENTS
}

Eureka

Volume 2, Number 1 (2011)

Lisa M. Guirguis \& Enoch Ng

Faculty of Pharmacy and Pharmaceutical Science, University of Alberta

\section{Introduction}

Little research has been conducted on empathy assessment in pharmacy education. The study objectives are to 1) compare pharmacy students' self-assessments and observer ratings of empathetic communication in simulations with standardized patients, 2) compare pharmacy students self-reported empathy with self-assessment and observer ratings, and 3) examine gender in empathetic communication.

\section{Methods}

First year pharmacy students enrolled in a communications course completed the empathic concern and perspective taking scales from the Interpersonal Reactivity Index (IRI) and participated in a video recorded patient interaction focusing on initial medication use. Students used the Empathic Communication Coding System (ECCS) to rate the first empathic opportunity in their video recording. Two researchers separately rated the video recordings using the ECCS for the empathic opportunity identified by the students and discrepancies were discussed until consensus was reached. Description statistics, correlations, and T-tests were used as appropriate to analyse the data.

\section{Results}

Analyses were performed on 63 students (class of 131) who consented and had complete data. Students rated themselves higher on the ECCS (3.6, SD=1.4) when compared with observers $(2.6, S D=1.1)(T$-test, $p<0.05)$. There were no statistically significant correlations between the IRI self-report and either student or observer ECCS ratings. Male students rated themselves lower on the ECCS $(3.2, S D=0.3)$ than females $(3.8, S D=0.8)$ while there were no gender differences in observer ECCS scores (male: 2.7, $\mathrm{SD}=1.5$; female: $2.6, \mathrm{SD}=1.3$ ).

\section{Discussion}

Pharmacy students were not able to accurately assess their own empathic communication. Our results indicate that students' self-report empathy was not correlated with behaviour. Observers found no difference between male and female empathetic communication; although males has lower self-assessment than their female colleagues. Further work is required to find appropriate methods for evaluating student empathy and developing accurate self-assessment of empathic communication.

Note: This abstract was presented at the European Association on Communication in Health Care in Verona, Italy from September 5-8, 2010. 